\title{
Functional analysis of Ectodysplasin-A mutations causing selective tooth agenesis
}

\author{
Gabriele Mues $^{1}$, Aubry Tardivel ${ }^{2}$, Laure Willen ${ }^{2}$, Hitesh Kapadia ${ }^{1}$, Robyn Seaman ${ }^{1}$, Sylvia Frazier-Bowers ${ }^{3}$, \\ Pascal Schneider ${ }^{2,4}$ and Rena N D’Souza ${ }^{\star, 1,4}$
}

\begin{abstract}
Mutations of the Ectodysplasin-A (EDA) gene are generally associated with the syndrome hypohidrotic ectodermal dysplasia (MIM 305100), but they can also manifest as selective, non-syndromic tooth agenesis (MIM300606). We have performed an in vitro functional analysis of six selective tooth agenesis-causing EDA mutations (one novel and five known) that are located in the C-terminal tumor necrosis factor homology domain of the protein. Our study reveals that expression, receptor binding or signaling capability of the mutant EDA1 proteins is only impaired in contrast to syndrome-causing mutations, which we have previously shown to abolish EDA1 expression, receptor binding or signaling. Our results support a model in which the development of the human dentition, especially of anterior teeth, requires the highest level of EDA-receptor signaling, whereas other ectodermal appendages, including posterior teeth, have less stringent requirements and form normally in response to EDA mutations with reduced activity.
\end{abstract}

European Journal of Human Genetics (2010) 18, 19-25; doi:10.1038/ejhg.2009.127; published online 22 July 2009

Keywords: Ectodysplasin-A mutations; EDAR; selective tooth agenesis; functional analysis

\section{INTRODUCTION}

Tooth development is largely under genetic control and all familial cases, as well as the majority of sporadic cases of congenital tooth agenesis are likely to be caused by sequence variations in one of the many genes that are involved in tooth development. ${ }^{1}$ Classically, a distinction has been made between non-syndromic and syndromic tooth agenesis. hypohidrotic ectodermal dysplasia (HED) is such a syndrome that features hypoplasia of most epithelial appendages including variable numbers of missing teeth, sparse hair and hypoplastic or aplastic sweat glands leading to hypohidrosis with potentially life-threatening hyperthermia. Non-syndromic tooth agenesis, on the other hand, has been defined as affecting predominantly the dentition and involving primarily the tooth-specific genes. ${ }^{2-4}$

The distinction between syndromic and non-syndromic tooth agenesis is becoming less apparent as genetic knowledge advances. Mutations in the Muscle Segment Homeobox 1 gene (MSX1), for example, not only cause selective tooth agenesis, but are also involved in orofacial cleft syndromes. ${ }^{5}$ Not only do the more 'tooth-specific' genes contribute to non-dental problems but also the reverse is observed: Several reports describe isolated tooth agenesis in families with mutations in Ectodysplasin-A (EDA), ${ }^{6-12}$ a gene that is usually responsible for the HED syndrome. ${ }^{13}$

The EDA gene occupies a 425-kb segment of the long arm of the $\mathrm{X}$-chromosome (Xq12-13.1) ${ }^{14}$ and encodes a protein that belongs to the tumor necrosis factor (TNF) superfamily of ligands. In total, eight isoforms of the EDA transcript can be created by differential splicing of the 12 exons. The longest splice form encodes a 391 amino acid protein with a transmembrane domain, a furin cleavage site, a collagen type repeat area in the middle and a C-terminal TNF-like structure. ${ }^{15-17}$ On proteolytic processing at the furin consensus site, the C-terminal portion of the protein containing the collagen domain and the TNF homology domain is released as a soluble, trimeric ligand. ${ }^{18}$ Only the following two splice isoforms of EDA contain the receptor-binding TNF homology domain: EDA1, which binds the EDA-receptor EDAR, and EDA2, a two amino acid shorter variant that binds exclusively to a receptor called XEDAR. ${ }^{19}$ The TNF homology domains of both EDA1 and EDA2 have been crystallized as homotrimers, but further multimerization through interactions of the collagen domain seem to be functionally important.

Both the receptors, EDAR and XEDAR, activate the NF- $\kappa$ B pathway, but only the EDA1/EDAR interaction seems relevant for the development of ectodermal appendages. Indeed, mutations in EDA1 as well as in EDAR can cause an HED syndrome that is phenotypically indistinguishable except for the different inheritance pattern, X-linked and autosomal, respectively. ${ }^{20}$ In addition, XEDAR mutations have not yet been identified in HED or any other condition, and XEDAR-deficient mice are normal and display none of the phenotypes characteristic of ectodermal dysplasia. $^{21}$

Almost a hundred different EDA gene mutations have been identified in patients with the HED syndrome. These mutations range from exon deletions and frameshifts to conservative replacements of single amino acids and may be located in any of the three main functional domains of the EDA protein, furin cleavage site, the collagen-like multimerization domain or the TNF homology domain. Phenotypically, the HED syndrome is diverse with a broad range of intra- and interfamilial variation in severity. However, despite the great number of different EDA mutations that have been studied so far, no genotype/ phenotype correlations have been uncovered.

\footnotetext{
${ }^{1}$ Department of Biomedical Sciences, Texas A\&M University Health Science Center, Baylor College of Dentistry, Dallas, TX, USA; ${ }^{2}$ Department of Biochemistry, University of Lausanne, Epalinges, CH, Switzerland; ${ }^{3}$ Department of Orthodontics, University of North Carolina at Chapel Hill, Chapel Hill, NC, USA

${ }^{\star}$ Correspondence: Professor RN D'Souza, Department of Biomedical Sciences, Texas A\&M University Health Science Center, Baylor College of Dentistry, 3302 Gaston Avenue, Dallas, TX 75246, USA. Tel: +1 214828 8375; Fax: +1 214874 4538; E-mail: rd'souza@bcd.tamhsc.edu ${ }^{4}$ These authors share senior authorship.

Received 1 May 2009; revised 18 June 2009; accepted 19 June 2009; published online 22 July 2009
} 
Previous investigations of the impact of EDA missense mutations on different aspects of protein function have shown that proteolytic processing, glycosylation, multimerization, and solubility or solely receptor binding can be affected by the different mutations. ${ }^{8,18,22}$ Ultimately, most of the mutations could be predicted to lead to an elimination of receptor signaling; only one mutation was shown to possess residual receptor-binding activity. Interestingly, this mutation was found in an HED family whose main complaint was tooth agenesis. ${ }^{8}$ Here, we describe an additional family with X-linked recessive, non-syndromic tooth agenesis that can be linked to an EDA mutation and present a functional analysis of this EDA mutation in comparison with other tooth agenesis and HED-causing mutations in the TNF homology domain of EDA.

\section{MATERIALS AND METHODS}

\section{Patient recruitment and phenotype evaluation}

The family participated in our IRB-approved tooth agenesis study. All adults consented to participate in this study; in the case of minors, parental consent and child assent in $>12$-year-olds were obtained. A pedigree was established showing that only males were affected. Phenotype evaluation was performed using an HED-specific questionnaire, interviews and panoramic radiographs of the dentition and/or dental records.

\section{Mutation analysis}

Blood samples or buccal swabs were collected for DNA extraction. A candidate gene approach was chosen with the X-chromosomal EDA gene as the first target. In total, eight exons of the EDA gene were PCR amplified and sequenced with automated fluorescent dideoxy technology.

A T-to-C transition in the last exon of the EDA gene of the affected index male was found to create an HhaI restriction site. All other participating members of the family as well as 65 unrelated females and 14 unrelated males were tested for the presence of this restriction site. Primers $5^{\prime}$ CACGCCTTCAC ATGGCACT3' and 5'CGGCTGCAACACCAATACAC3' were used for amplification of the exon.

\section{Construction of EDA expression vectors}

Mammalian expression vectors for secreted Flag-tagged forms of EDA1 and EDA2 with the tooth agenesis-causing mutations V365A, Q358E, D316G, T338M or M364T were generated as described earlier for other EDA mutants, including S374R. ${ }^{8}$ Briefly, these constructs code for the signal peptide of hemagglutinin, the Flag sequence (DYKDDDDK), a linker (GPGQVQLQVD) and the TNF homology domain of EDA1 (amino acids 245-391) or EDA2 (amino acids 245-389). Other EDA constructs used in this study and expression vectors for human EDAR-Fc and human XEDAR-Fc have been described earlier. $^{8}$

\section{Expression of soluble EDA in cell supernatants}

Vectors encoding soluble, wild-type (wt) or mutant Flag-tagged EDA1 or EDA2 protein, or EDAR:Fc were transfected into $293 \mathrm{~T}$ cells, and the cells were maintained in serum-free OptiMEM (Invitrogen Corporation, Carlsbad, CA USA) for 7 days. The concentration of Flag-tagged EDA proteins in cell supernatants was estimated by comparative anti-Flag Western blot using serial dilutions of each protein and known amounts of purified Flag-TRAIL.

\section{Efficiency of EDA secretion}

293 T cells were transfected with wild-type or mutant Flag-EDA1, and supernatant and cells were harvested separately. The cell pellets were lysed by sonication in a known volume of SDS-PAGE sample buffer. Two-fold serial dilutions of cell extracts and the corresponding supernatants were evaluated for relative EDA protein content by densitometry of slot blots developed with antiFlag. The percentage of secretion was calculated as (EDA amount in supernatant) divided by (EDA amount in cells + supernatant $) \times 100$.

\section{ELISA assay for receptor-ligand binding}

The binding of wild-type and mutant EDA1 and EDA2 to EDAR and XEDAR was monitored by ELISA as described earlier. ${ }^{8,23}$ Briefly, ELISA plates were coated with a mouse anti-human monoclonal antibody ( $100 \mu$ la a concentration of $5 \mu \mathrm{g} / \mathrm{ml}$ in $50 \mathrm{~mm}$ of $\mathrm{Na}$ carbonate ( $\mathrm{pH}$ 9)), blocked, incubated with $100 \mu \mathrm{l}$ of EDAR:Fc or XEDAR:Fc (Alexis, Lausen, Switzerland) at a concentration of $0.5 \mu \mathrm{g} / \mathrm{ml}$, followed by two-fold dilutions of quantified Flag-EDA. Bound ligands were revealed with biotinylated anti-Flag antibody $(0.5 \mu \mathrm{g} / \mathrm{ml}$, Sigma-Aldrich, Buchs, Switzerland), horseradish peroxidase-conjugated streptavidin (1/4000, Jackson Immunoresearch, Newmarket, UK) and orthophenylene diamine reagent, and read at $492 \mathrm{~nm}$.

\section{Generation of EDAR:Fas Jurkat cells}

Fas-deficient Jurkat-JOM2 cells expressing the EDAR:Fas fusion protein were generated essentially as described earlier for BCMA:Fas. ${ }^{24}$ For this purpose, the retroviral vector pMSCVpuro encoding the extracellular domain of human EDAR (amino acids 1-183), amino acids VD and the transmembrane, and intracellular domains of human Fas (amino acids 169-335) was used. About 40 clones were tested for their sensitivity to $\mathrm{Fc}-\mathrm{EDAl},{ }^{25}$ and one of the sensitive clones (Jurkat-2199 clone 23) was selected for further experimentation. In these cells, the binding of EDA1 to EDAR:Fas induces cell death through the Fas signaling pathway. Cytotoxicity assays with Flag-EDA1 wt or mutants were performed in the presence or absence of $2 \mu \mathrm{g} / \mathrm{ml}$ of cross-linking anti-Flag M2 monoclonal antibody (Sigma) as described earlier. ${ }^{26,27}$

\section{RESULTS}

Mutation detection in a tooth agenesis family

The pedigree of this Caucasian tooth agenesis family shows a typical $\mathrm{X}$-linked recessive pattern of inheritance, affecting only males (Figure 1a). Figure 1b gives an overview of the dental phenotype of the affected participants. In all cases, two to six primary or permanent incisors are missing, with the exception of maxillary central incisors. First or second premolars and second molars of the permanent dentition may be missing as well. Carrier female family members report full dentition without shape or size abnormality.

As the pedigree pattern was strongly suggestive of an X-linked condition and the EDA gene on Xq12-13.1 is associated with a syndrome that includes tooth agenesis, a candidate gene approach was pursued and led to the detection of a $\mathrm{T} \rightarrow \mathrm{C}$ transition at nucleotide 1336 of the deduced cDNA sequence. The nucleotide replacement produces a conservative change of the surface-expressed Valine 365 to Alanine (Figure $2 \mathrm{a}$ and $\mathrm{b}$ ). The $\mathrm{T} \rightarrow \mathrm{C}$ nucleotide change also introduces an HhaI restriction site into the last exon of the EDA gene, which allowed the testing of all 26 available family members for the V365A mutation by restriction fragment length polymorphism. A perfect segregation of the T-to-C transition with the phenotype became apparent: Affected males have a single mutant copy, carrier females have one mutant and one wild-type copy, and unaffected probands have only wild-type copy(s) (Figure 1a).

A common polymorphism at this site of the EDA gene was excluded by testing 79 controls with a total of $144 \mathrm{X}$-chromosomes ( 65 females, 14 males) for the T-to-C transition at nucleotide 1336. None of these unrelated controls showed the mutation. We conclude that the V365A substitution is the likely cause for the tooth agenesis in this family.

To evaluate the phenotype further, participating family members were asked to complete a detailed two-page questionnaire (summarized in Table 1). The only symptoms reminiscent of HED seem to be a tendency for fine, curly hair, early balding (in their middle to late twenties) and thin skin. Heat intolerance is reported not only by some of the affected males but also by unaffected family members. The condition has been observed for four generations in this family but was never diagnosed as ectodermal dysplasia because of the 
a
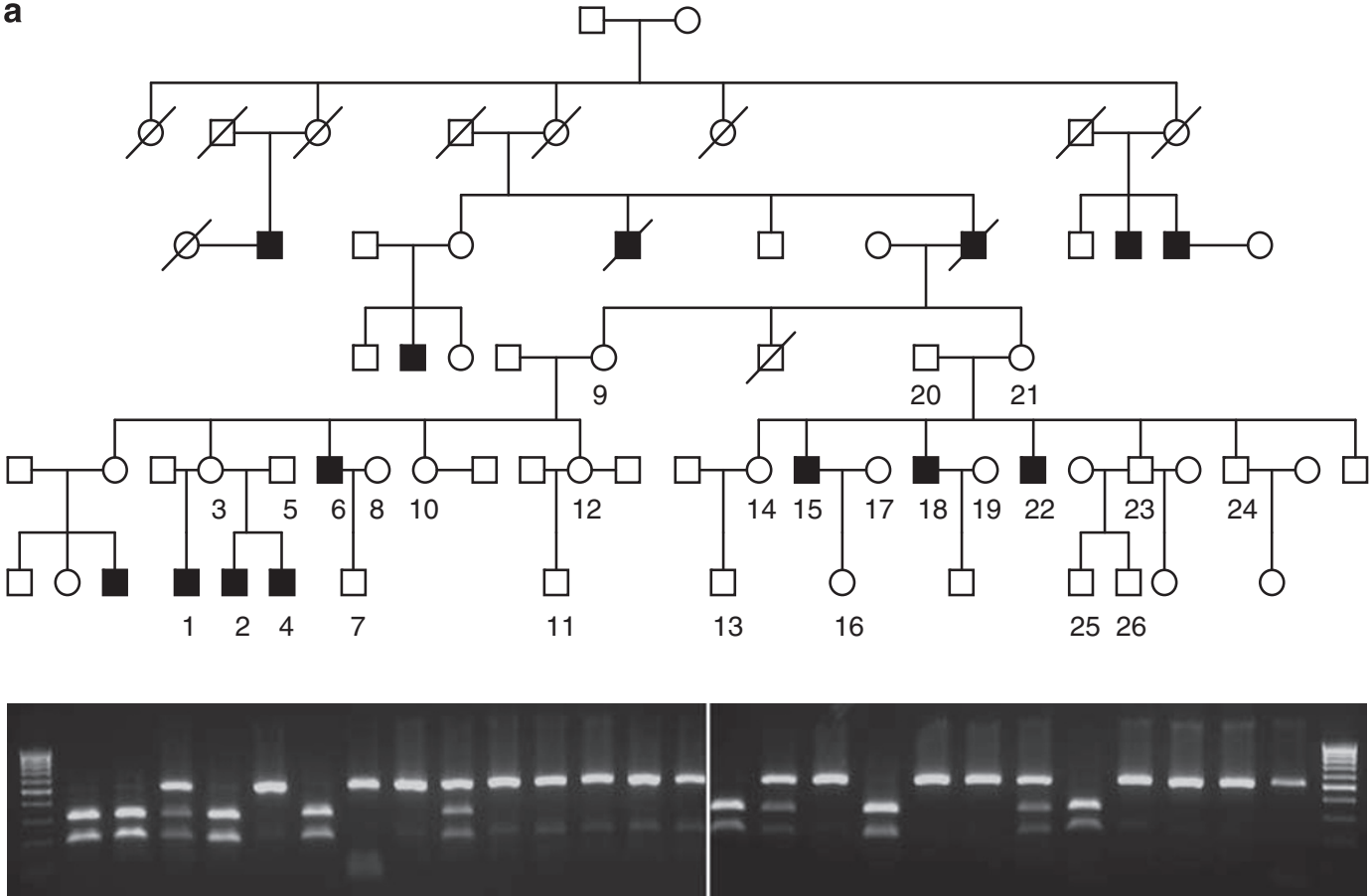

$\begin{array}{llllllllllllllllllllllllllll}\text { Std } & 1 & 2 & 3 & 4 & 5 & 6 & 7 & 8 & 9 & 10 & 11 & 12 & 13 & 14 & 15 & 16 & 17 & 18 & 19 & 20 & 21 & 22 & 23 & 24 & 25 & 26 & \text { Std }\end{array}$

b

\begin{tabular}{|c|c|c|c|c|c|c|c|c|c|c|}
\hline \multirow[t]{2}{*}{1} & 5 & 4 & 3 & & 1 & 1 & & 3 & 4 & 5 \\
\hline & 5 & 4 & 3 & 2 & 1 & 1 & 2 & 3 & 4 & 5 \\
\hline \multirow[t]{2}{*}{2} & 5 & 4 & 3 & & 1 & 1 & & 3 & 4 & 5 \\
\hline & 5 & 4 & 3 & 2 & 1 & 1 & 2 & 3 & 4 & 5 \\
\hline \multirow[t]{2}{*}{4} & 5 & 4 & 3 & & 1 & 1 & & 3 & 4 & 5 \\
\hline & 5 & 4 & 3 & 2 & 1 & 1 & 2 & 3 & 4 & 5 \\
\hline \multirow[t]{2}{*}{6} & 5 & 4 & 3 & & 1 & 1 & & 3 & 4 & 5 \\
\hline & 5 & 4 & 3 & 2 & 1 & 1 & 2 & 3 & 4 & 5 \\
\hline \multirow[t]{2}{*}{15} & 5 & 4 & 3 & & 1 & 1 & & 3 & 4 & 5 \\
\hline & 5 & 4 & 3 & & & & & 3 & 4 & 5 \\
\hline \multirow[t]{2}{*}{18} & 5 & 4 & 3 & & 1 & 1 & & 3 & 4 & 5 \\
\hline & 5 & 4 & 3 & & & & 2 & 3 & 4 & 5 \\
\hline \multirow[t]{2}{*}{22} & 5 & 4 & 3 & 2 & 1 & 1 & & 3 & 4 & 5 \\
\hline & 5 & 4 & 3 & & & & 2 & 3 & & 5 \\
\hline
\end{tabular}

Permanent teeth

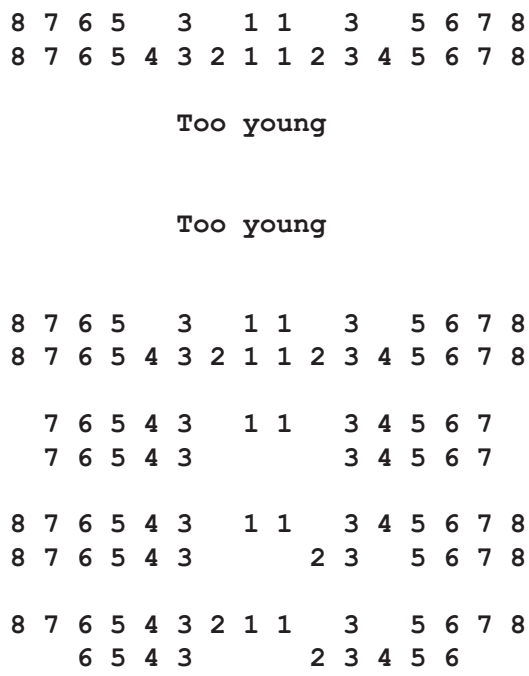

Figure 1 Pedigree and phenotype of a family with selective tooth agenesis. (a) Pedigree of the family with non-syndromic tooth agenesis and segregation of the Hha1 restriction site (created by the T-to-C transition in the Ectodysplasin-A (EDA) gene) with affected and carrier status. Numbers 1-26 denote the family members for whom DNA samples were available. Open circles, healthy female; open squares, healthy male; filled squares, affected male; /, deceased. (b) Tooth agenesis pattern in affected males. Incisors are most frequently absent, in particular mandibular incisors. Maxillary central incisors are rarely affected in this Caucasian family, consistent with previous reports ${ }^{29}$. Primary teeth: 1 , central incisors; 2 , lateral incisors; 3 , canines; 4-5, molars and permanent teeth: 1 , central incisors, 2 , lateral incisors, 3, canines, 4-5, premolars; 6-8, molars.

uncharacteristically mild systemic features that are not significantly different from random variability in the general population. We conclude that this family's features fit the designation, X-linked Hypodontia MIM300606.

\section{Functional characterization of mutant protein constructs}

The V365A mutation and the five other mutations ${ }^{8-12}$ that cause selective tooth agenesis are located in the $\mathrm{COOH}$-terminal quarter of the TNF-like receptor-binding domain (Figure 2a), and cluster close to the interface of two adjacent monomers where receptor binding is predicted to occur ${ }^{23}$ (Figure 2b).

All six mutations were tested for expression, glycosylation, cellular export, quantitative receptor binding and signaling ability as described earlier for the S374R mutation. ${ }^{8}$ Figure 3a shows normalized amounts of wild-type and mutant EDA1 and EDA2, with the tooth agenesiscausing mutations V365A, Q358E, S374R, D316G, T338M and 

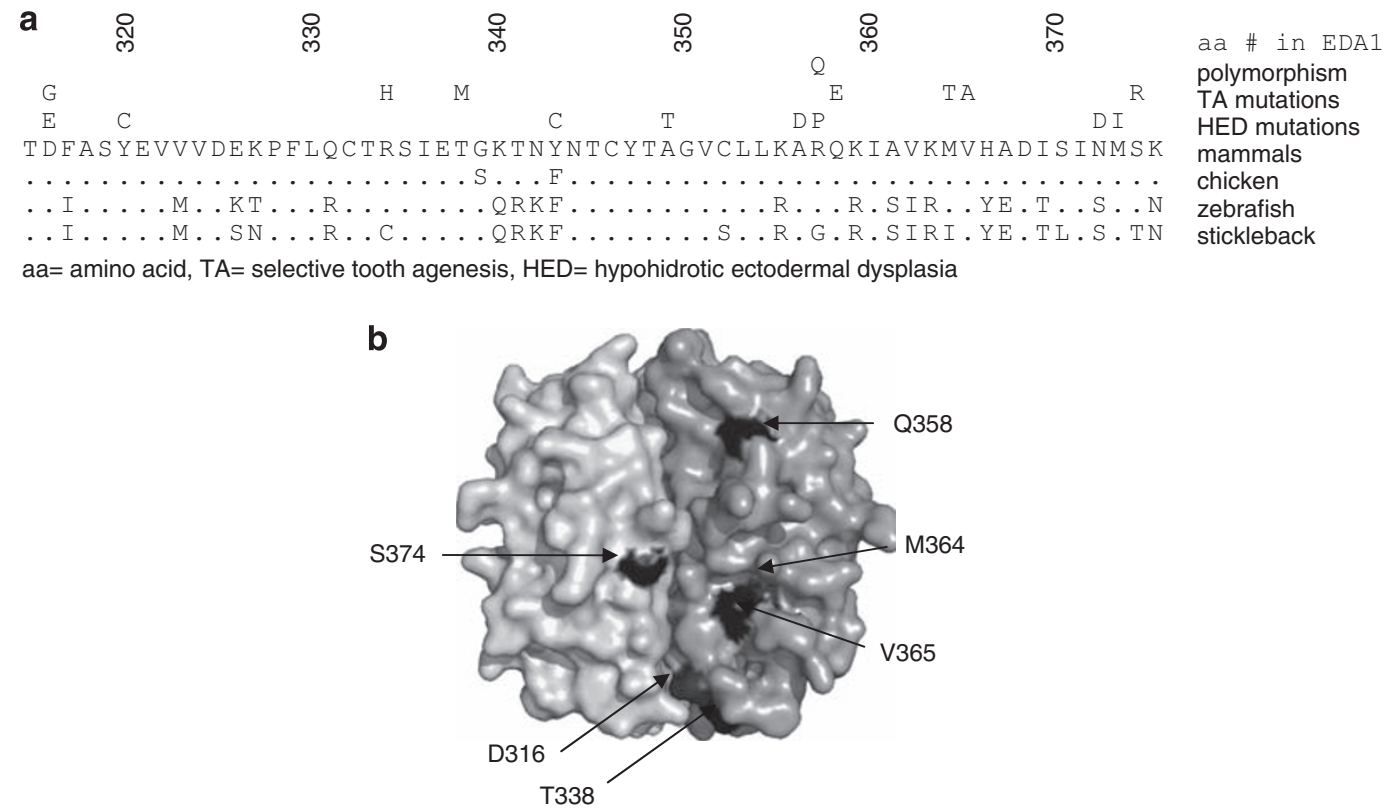

Figure 2 Amino acid comparison of Ectodysplasin-A (EDA) proteins from different species and position of the tooth agenesis-causing mutations. (a) Alignment of a portion of the TNF homology domain of EDA from different species, showing the locations of V365A and five further missense mutations causing isolated tooth agenesis (D316G, T338M, Q358E, M364T and S374R). The EDA amino acid sequences for human, chimp, rhesus, cow, dog, rat and mouse are identical in this region and are referred to as 'mammals.' Numbering corresponds to the human sequence. The positions of some hypohidrotic ectodermal dysplasia (HED)-causing mutations and of the only known polymorphism are also shown. (b) Tooth agenesis-causing EDA mutations are surfacelocated in the predicted receptor-binding region at the interface of two protomers. The figure is based on the PDB atomic coordinate file 1 RJ7.

Table 1 Frequency of HED-like symptoms in seven affected males, five carrier females and eight unaffected family members

\begin{tabular}{lccc}
\hline & Affected (7) & Carrier (5) & Unaffected (8) \\
\hline Sparse, thin hair & $1 / 7$ & $0 / 5$ & $0 / 8$ \\
Early balding $^{\text {a }}$ & $1 / 4$ & $0 / 5$ & $0 / 8$ \\
Thin skin & $1 / 7$ & $0 / 5$ & $0 / 8$ \\
Overheating easily & $2 / 7$ & $1 / 5$ & $3 / 8$ \\
High childhood fevers & $0 / 7$ & $1 / 5$ & $2 / 8$ \\
\hline
\end{tabular}

HED, hypohidrotic ectodermal dysplasia.

aOnly adults are counted.

These symptoms have been rated as 'clearly present or frequently a problem'

M364T, or the HED-causing mutations Y343C and H252L. The two bands in each lane represent the glycosylated and the unglycosylated polypeptides of each construct, as shown by peptide-N glycanase $\mathrm{F}$ digestion (Figure 3b). Although all nine protein constructs are expressed, glycosylated and exported out of the cells, there are a few marked differences. In mutant Q358E, the glycosylated band is consistently overrepresented when compared with the lower nonglycosylated band. This may indicate a solubility problem of the unglycosylated form, causing it to be retained in the cell. Indeed, $<10 \%$ of the tooth agenesis-causing mutant Q358E is recovered from cell supernatants. Secretion problems are not detectable in the other mutants, with the exception of the HED-causing Y343C variant, which is also mostly retained in the transfected cells, consistent with our previous report ${ }^{8}$ (Figure $2 \mathrm{~d}$ ).

The relative amounts of Flag-tagged wt and mutant proteins were carefully determined to exclude the fact that the small differences in receptor-binding activity are due to a difference in ligand concentration
(Figure 3c). Indeed, the quantitative ELISA (Figure 4a, upper panel) shows that the binding of all hypodontia-causing EDA1 proteins to the receptor EDAR is variably reduced compared with wt, but not abolished. In contrast, EDA1 carrying either of the two HEDcausing mutations $\mathrm{Y} 343 \mathrm{C}$ and $\mathrm{H} 252 \mathrm{~L}$ lose all receptor-binding capability, consistent with the results that we obtained earlier by testing HED-causing mutants. ${ }^{8}$ The affinity of the corresponding EDA2 proteins to XEDAR is only slightly reduced, if reduced at all, for five of the six tooth agenesis-causing mutants (Figure $4 \mathrm{a}$, lower panel).

We next investigated the ability of EDA1 wt and mutant constructs to trigger a surrogate, multimerization-dependent signaling pathway in Jurkat $\mathrm{T}$ cells engineered to express an EDAR:Fas fusion receptor. In this system, a functional EDA1 ligand can induce cell death through the Fas pathway; however, this requires earlier cross-linking of the EDA1 ligand with anti-Flag antibodies in analogy to the cross-linking of FasL, which is required for signaling in the original FasL/Fas pathway. ${ }^{26}$ Figure $4 \mathrm{~b}$ shows that cross-linked wt EDA1 can induce cell death in this assay. The Q358E mutant is the only one showing appreciable yet reduced activity in this assay. Surprisingly, V365A and M364T are mostly inefficient in the cell death-induction assay, indicating that the signaling capacity of the mutated proteins is impaired even though cellular production, export and receptor binding are readily detectable. S374R and T338M have hardly any activity in the EDAR-Fas signaling assay, in line with their severely reduced receptor-binding capacity. Our assays would predict the D316G mutation to have the most severe effect, and according to the published phenotype data, this seems to be the case. ${ }^{9}$

\section{DISCUSSION}

The development of teeth depends on a highly coordinated interaction of multiple signaling events between ectodermal and underlying 
a

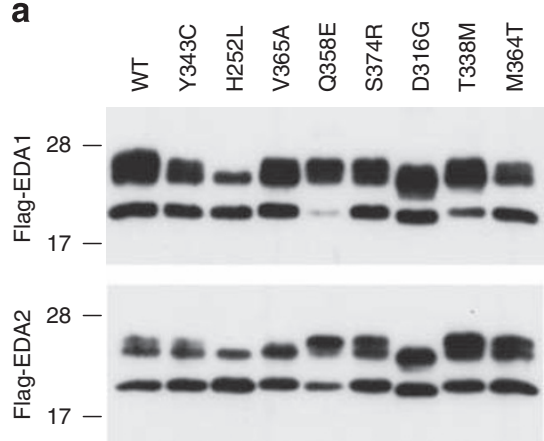

C

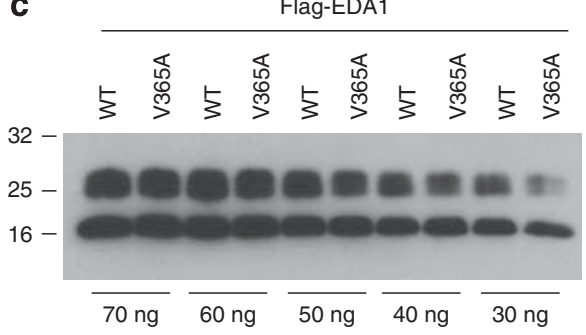

b
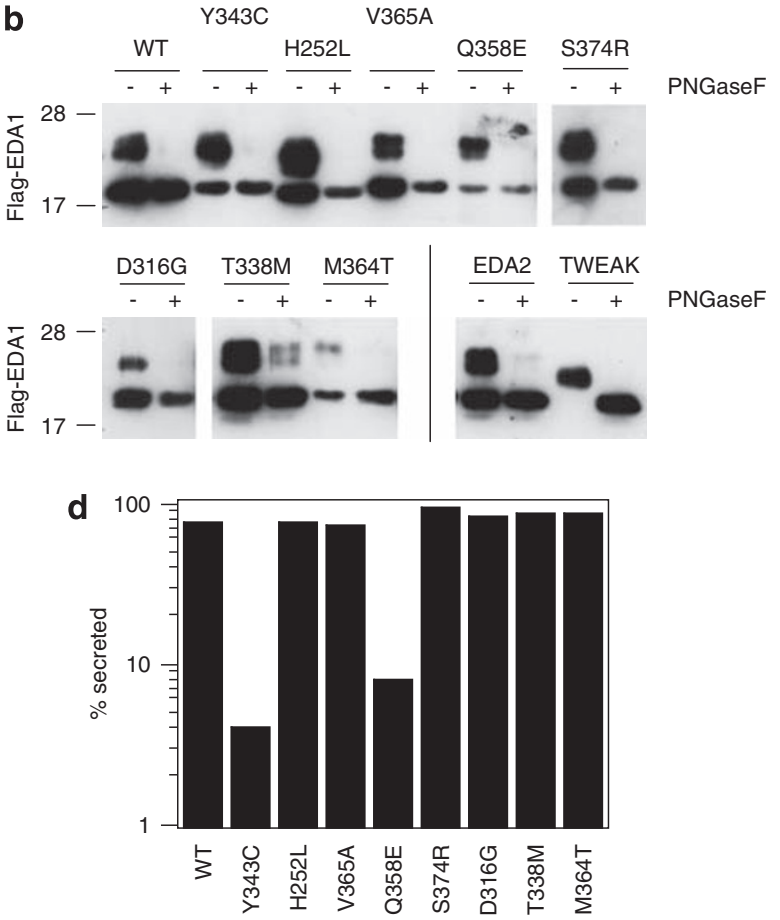

Figure 3 (a) Normalized amounts of wild-type and mutant Flag-EDA1 (upper panel) or Flag-EDA2 proteins (lower panel) from supernatants of transfected 293T cells were analyzed by anti-Flag western blot. (b) Flag-EDA1 (wild-type and indicated mutants), Flag-EDA2 (wild type) and the positive control Flag-TWEAK were treated with or without peptide-N-glycanase F (PNGaseF) and analyzed by anti-Flag western blot. (c) Similar amounts of Flag-EDA1 wt and V365A were analyzed by anti-Flag western blot to validate the estimation of protein concentration. (d) Flag-EDA1 present in cell extracts and cell supernatants were quantified to estimate secretion efficiency. All constructs contain the signal peptide of hemagglutinin. Protein secretion is consistently impaired by the tooth agenesis-causing mutation, Q358E, and the hypohidrotic ectodermal dysplasia (HED)-causing mutation, Y343C.

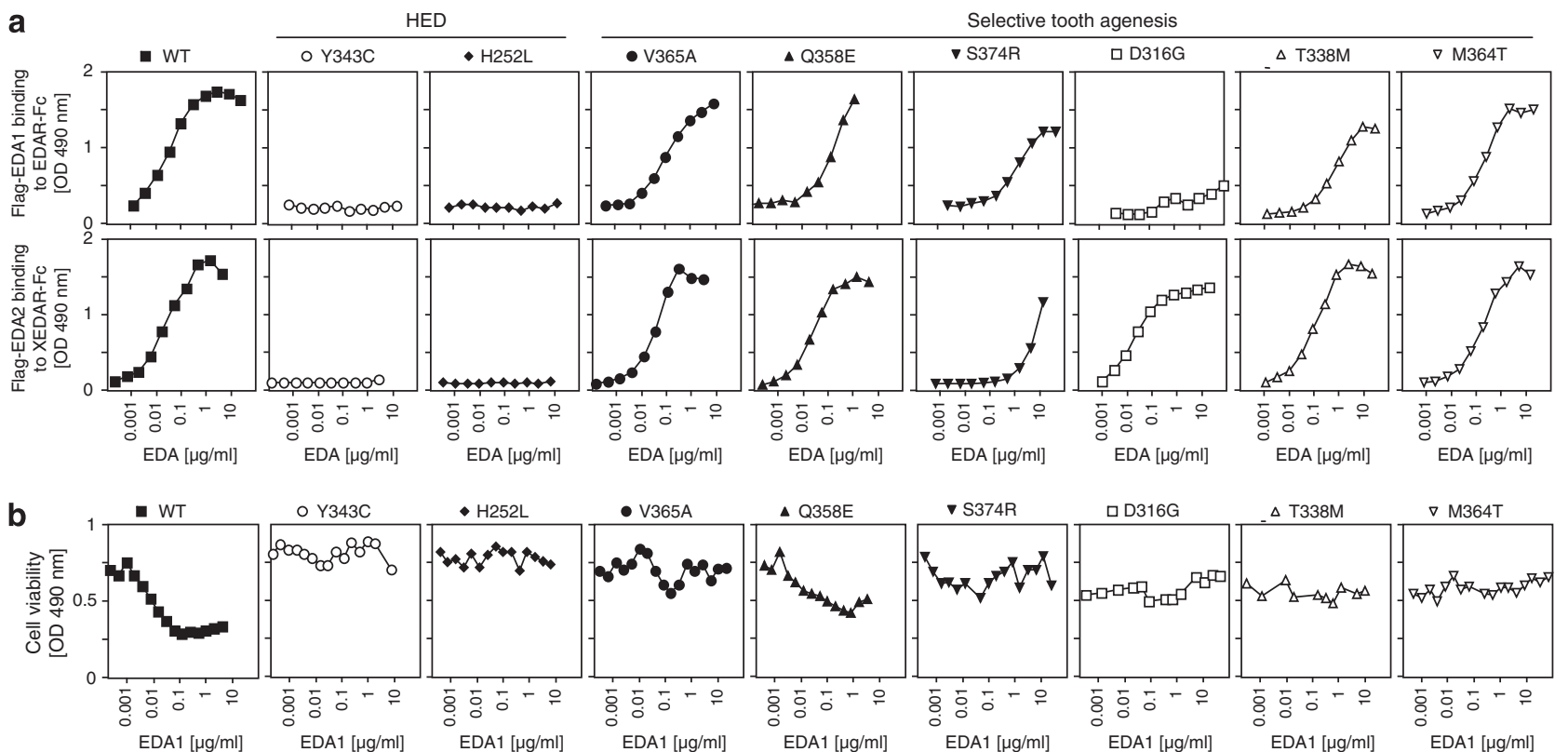

Figure 4 (a) Quantitative binding of EDA1 to EDAR (upper panel) and EDA2 to XEDAR (lower panel), as measured by ELISA. All six tooth agenesis-causing mutants retain residual receptor binding to EDAR in contrast to the hypohidrotic ectodermal dysplasia (HED)-causing mutants Y343C and H252L, which lose all receptor-binding ability. Binding of EDA2 to XEDAR is not or only moderately affected for five of the six tooth agenesis-causing mutants. The results are representative of three to six experiments (depending on the mutant) with similar results. (b) EDAR:Fas-expressing Jurkat cells were treated with the indicated concentrations of wt and mutant EDA1 preparations. The surrogate apoptotic Fas pathway is monitored as reduced cell viability using the PMS/MTS reagents. Successful activation occurs only in the presence of a cross-linking anti-Flag antibody. Only wild-type EDA1 and mutant Q358E were significantly active in this assay. The results are representative of three independent experiments with similar results. 
mesenchymal cells, similar to the development of other skin appendages such as hair follicles, nails and glands. A multitude of genes are known to be expressed in the developing tooth, but so far only four of them have been associated with selective, non-syndromic tooth agenesis in humans (1) The Paired Box 9 gene (PAX9) is strongly expressed in developing teeth. Heterozygous mutations in PAX9 cause predominantly molar tooth agenesis. (2) MSX1, a homeobox protein, can cause a slightly different phenotype with more premolar involvement. It may also contribute to Witkop ${ }^{28}$ and cleft lip/palate syndromes. (3) Axis Inhibitor 2 (AXIN2) is a signaling molecule in the 'wingless-type MMTV integration site family' (WNT) pathway that starts operating very early in tooth placode formation. Autosomal dominant mutations in exon 7 of AXIN2 have been shown to cause not only a severe mixed pattern of tooth agenesis but also intestinal polyposis and predisposition to colon cancer. (4) The EDA gene, known to be affected in most patients with the HED syndrome, is also implicated in selective tooth agenesis. The hypodontia phenotype in EDA-associated isolated tooth agenesis seems to favor the lack of incisors, but other teeth are involved as well.

However, these four genes are responsible for tooth agenesis in only a fraction of all observed hypodontia cases. Many more genes remain to be discovered; and so far, only a few functional analyses of tooth agenesis-causing mutations are available to explain the pathogenesis of congenital hypodontia.

Most of the known EDA mutations affect a variety of ectodermal appendages such as hair, teeth and glands. Absent or severely decreased sweating can lead to hyperthermia and death during minor febrile infections in childhood. Other problems caused by glandular hypoplasia are constipation, respiratory infections, insufficient production of saliva and lacrimal fluid, earwax impaction, frequent middle-ear infections, and underdeveloped mammillae and lactation difficulties in affected females. Hair and eyebrows are sparse or missing. Some affected males lack all teeth, and some only a few, but on an average 22 permanent teeth are missing, not counting third molars. ${ }^{29}$ Shape abnormalities include smaller size, peg shape, reduced cusp number and root abnormalities. The facial features may be slightly dysmorphic with frontal bossing and depressed nasal bridge.

The HED often manifests with variability among affected males of the same family. Carrier females generally show a much milder phenotype with only one or two teeth missing, slightly smaller teeth, sparse hair or no symptoms at all. Mutations in all portions of the gene can cause ectodermal dysplasia, with no apparent correlation between the location of the mutation and severity of the phenotype.

The V365A mutation reported here is located in the C-terminal TNF-like portion of the protein, as are most of the other tooth agenesis-causing mutations, including the three newly-described ones (A259E, R289C and R334H). ${ }^{30}$ However, this position is not required for the milder effect of these mutations. One selective tooth agenesiscausing EDA mutation, G165A, is located at the end of the collagenlike domain; ${ }^{31}$ two others $\left(\mathrm{R}_{65} \mathrm{G}^{6}\right.$ and $\mathrm{E} 67 \mathrm{~V}^{12}$ ) are located at the extracellular end of the EDA transmembrane sequence. The R65G and E67V replacements may reduce the expression or translocation, and therefore the dosage of otherwise normal ligands. In HED patients, this area is less frequently affected, and the predominance of frameshift and nonsense mutations suggests that the importance of mutations occurring in this part lies more in the abolishment of the rest of the protein than in a local dysfunctional effect. On the other hand, amino acid changes in this least conserved region of EDA are responsible for major adaptive changes in stickleback evolution ${ }^{32}$ and may have some other important function.
In our previous functional analysis of HED causing EDA mutations, ${ }^{8}$ we noticed that the only mutation with residual receptor-binding activity, S374R, came from a family with a predominantly missing teeth phenotype. However, we could not determine whether this was a coincidence or a truly tissue-specific phenomenon. Our current results strongly suggest that there is indeed a tissue-specific effect, in that residual EDA/EDAR activity can prevent the hypoplasia of most ectodermal appendages, except for the development of incisors and a few other teeth. In other words, the development of teeth, especially of incisors and premolars, requires the highest dosage of EDA signaling. However, the mutations associated with tooth agenesis are remarkably dissimilar in the effects they produce in assays with recombinant EDA. The adjacent mutations V365A and M364T are well expressed and show modestly lower binding to EDAR, which translates into a major impairment at activating a cross-linking-dependent surrogate signaling pathway.

For S374R, we reproduce the weak binding of the EDA1 isoform to EDAR, ${ }^{8}$ but see little, if any, activity in the bioassay. We also observe a much reduced but detectable binding of the EDA2 isoform of this mutant to XEDAR. The mutant proteins T338M and D316G share similar functional properties such as S374R with D316G performing worst in the EDAR-binding assay and D316G family members presenting with anodontia or severe oligodontia. Mutation Q358E has different effects. Binding to XEDAR and EDAR is little affected, and it retains significant capacity to activate multimerization-dependent signal transduction. However, the $90 \%$ reduced secretion level may explain the observed phenotype.

Altogether, we propose that EDA mutations associated with selective tooth agenesis share the property to retain some receptor signaling activity, but to be impaired either in receptor binding, and/or in their expression level and/or in their ability to induce efficient signaling downstream of the receptor. The phenotype strongly suggests that the threshold for EDA signaling in tooth development must be higher than in other EDA-dependent structures. Tooth shape abnormalities seem to be less frequent in EDA-caused selective tooth agenesis, possibly reflecting different requirements for the level of EDA/ EDAR activity in shape formation. There are other examples in which different levels of residual gene function can lead to sometimes dramatically different clinical phenotypes, which are as follows: More severe mutations in the X-chromosomal NEMO gene, which operates downstream in the EDA/EDAR signaling pathway, lead to incontinentia pigmenti in females and prenatal lethality in males. Milder mutations present as HED with severe immunodeficiency in surviving males. ${ }^{33}$

The finding that tooth development requires more EDA pathway signaling than other ectodermal appendages is supported by two observations made in EDA-deficient animals treated with a recombinant form of EDA1 endowed with cross-placental transfer properties by fusion to the Fc portion of IgG1 (Fc-EDA1). When Fc-EDA1 was administered to pregnant EDA-deficient (Tabby) mice, most Tabbyrelated defects were permanently rescued in offspring, except for tooth number and a special type of murine hair. ${ }^{25}$ In the second experiment, recombinant EDA preparations were injected postnatally into dog pups with the HED syndrome. It was recognized that the development of permanent teeth required a relatively higher or repeated dosage of recombinant EDA-A1. ${ }^{34}$ These findings also point to the exciting possibility that the isolated tooth agenesis of permanent teeth caused by EDA mutations may be preventable in humans. Health care professionals should be aware of the possibility that patients with agenesis of incisors and various other anterior teeth may carry a 
mutation in the EDA gene without showing other classical ectodermal dysplasia symptoms.

\section{CONFLICT OF INTEREST}

The authors declare no conflict of interest.

\section{ACKNOWLEDGEMENTS}

We thank the tooth agenesis family for their cooperation, Joseph F Maher, MD, for advice with the EDA questionnaire, and Sarah C Pollan White, DDS, for orthodontic evaluations. This work was supported by National Institute of Health Grant U24-PEI6472 to RDS and by Swiss National Science Foundation Grant 3100A0-122553 to PS.

1 Thesleff I: Epithelial-mesenchymal signaling regulating tooth morphogenesis. J Cell Sci 2003; 116: 1647-1648.

2 Vastardis H, Karimbux N, Guthua SW, Seidman JG, Seidman C: A human MSX1 homeodomain missense mutation causes selective tooth agenesis. Nat Genet 1996; 13: 417-421.

3 Stockton DW, Das P, Goldenberg M, D'Souza RN, Patel PI: Mutation of PAX9 is associated with oligodontia. Nat Genet 2000; 24: 18-19.

4 Lammi L, Arte S, Somer M et al: Mutations in AXIN2 cause familial tooth agenesis and predispose to colorectal cancer. Am J Hum Genet 2004; 74: 1043-1050.

5 Van den Boogaar M-JH, Dorland M, Beemer FA, Ploos van Amstel HK: MSX1 mutation is associated with orofacial clefting and tooth agenesis in humans. Nat Genet 2000; 24: 342-343. Erratum: Nat Genet 2000; 25: 125.

6 Tao R, Jin B, Guo SZ et al: A novel missense mutation of the EDA gene in a Mongolian family with congenital hypodontia. J Hum Genet 2006; 51: 498-502.

7 Tarpey P, Pemberton TJ, Stockton DW et al: A novel Gln358Glu mutation in ectodysplasin-A associated with X-linked dominant incisor hypodontia. Am J Med Genet 2007; 143A: 390-394.

8 Schneider P, Street SL, Gaide 0 et al: Mutations leading to X-linked hypohidrotic ectodermal dysplasia affect three major functional domains in the tumor necrosis factor family member ectodysplasin-A. J Biol Chem 2001; 276: 18819-18827.

$9 \mathrm{Li} \mathrm{S}, \mathrm{Li} \mathrm{J}$, Cheng J et al: Non-syndromic tooth agenesis in two Chinese families associated with novel missense mutation in the TNF domain of EDA (ectodysplasin A). PLOS ONE 2008; 3: e2396.

10 Han D, Gong Y, Wu $\mathrm{H}$ et al: Novel EDA mutation resulting in X-linked non-syndromic hypodontia and the pattern of EDA-associated isolated tooth agenesis. Eur $\mathrm{J}$ Med Genet 2008; 51: 536-546.

11 Rasool M, Schuster J, Aslam M et al: A novel missense mutation in the EDA gene associated with X-linked recessive isolated hypodontia. J Hum Genet 2008; 53: 894-898.

12 Fan $\mathrm{H}$, Ye X, Shi L et al: Mutations in the EDA gene are responsible for X-linked hypohidrotic ectodermal dysplasia and hypodontia in Chinese kindreds. Eur J Oral Sci 2008; 116: 412-417.

13 Kere J, Srivastava AK, Montonen 0 et al: X-linked anhidrotic (hypohidrotic) ectodermal dysplasia is caused by mutation in a novel transmembrane protein. Nat Genet 1996; 13: 409-416.

14 Zonana J, Clarke A, Sarfarazi M et al: X-linked hypohidrotic ectodermal dysplasia: localization within the region $\mathrm{Xq11-21.1}$ by linkage analysis and implications for carrier detection and prenatal diagnosis. Am J Hum Genet 1988; 43: 75-85.
15 Bayés M, Hartung AJ, Ezer $\mathrm{S}$ et al: The anhidrotic ectodermal dysplasia gene (EDA) undergoes alternative splicing and encodes ectodysplasin-A with deletion mutations in collagenous repeats. Hum Mol Genet 1998; 7: 1661-1669.

16 Monreal A, Zonana J, Ferguson B: Identification of a new splice form of the EDA1 gene permits detection of nearly all $\mathrm{x}$-linked hypohidrotic ectodermal dysplasia mutations. Am J Hum Genet 1998; 63: 380-389.

17 Ezer S, Bayés M, Elomaa O, Schlessinger D, Kere J: Ectodysplasin is a collagenous trimeric type II membrane protein with a tumor necrosis factor-like domain and colocalizes with cytoskeletal structures at lateral and apical surfaces of cells. Hum $\mathrm{Mol}$ Genet 1999; 8: 2079-2086.

18 Elomaa O, Pulkkinen K, Hannelius U, Mikkola M, Saarialho-Kere U, Kere J: Ectodysplasin is released by proteolytic shedding and binds to the EDAR protein. Hum Mol Genet 2001; 10: 953-962.

19 Yan M, Wang LC, Hymowitz SG et al: Two-amino acid molecular switch in an epithelial morphogen that regulates binding to two distinct receptors. Science 2000; 290: 523-527.

20 Monreal A, Ferguson BM, Headon DJ, Street SL, Overbeek PA, Zonana J: Mutations in the human homologue of mouse $\mathrm{dl}$ cause autosomal recessive and dominant hypohidrotic ectodermal dysplasia. Nat Genet 1999; 22: 366-369.

21 Newton K, French DM, Yan M, Frantz GD, Dixit VM: Myodegeneration in EDA-A2 transgeneic mice is prevented by XEDAR deficiency. Mol Cell Biol 2004; 24: 1608-1613.

22 Chen Y, Molloy SS, Thomas L et al: Mutations within a furin consensus sequence block proteolytic release of ectodysplasin-A and cause X-linked hypohidrotic ectodermal dysplasia. PNAS 2001; 98: 7218-7223.

23 Thompson JS, Bixler SA, Qian F et al: BAFF-R, a newly identified TNF receptor that specifically interacts with BAFF. Science 2001; 293: 2108-2111.

24 Bossen C, Cachero TG, Tardivel A et al: Interactions of tumor necrosis factor (TNF) and TNF receptor family members in the mouse and human. Blood 2008; 111: 1004-1012.

25 Gaide 0, Schneider P: Permanent correction of an inherited ectodermal dysplasia with recombinant EDA. Nat Med 2003; 9: 614-618.

26 Schneider P, Holler N, Bodmer JL et al: Conversion of membrane-bound FAS (CD95) ligand to its soluble form is associated with downregulation of its proapoptotic activity and loss of liver toxicity. J Exp Med 1998; 187: 1205-1213.

27 Holler N, Tardivel A, Kovacsovics-Bankowski M et al: Two adjacent trimeric FAS ligands are required for FAS signaling and formation of a death-inducing signaling complex. Mol Cell Biol 2003; 23: 1428-1440.

28 Jumlongras D, Bei M, Stimson JM et al: A nonsense mutation in MSX1 causes Witkop syndrome. Am J Hum Genet 2001; 69: 67-74.

29 Lexner MO, Bardow A, Hertz JM, Nielsen LA, Kreiborg S: Anomalies of tooth formation in hypohidrotic ectodermal dysplasia. Internat J Paed Dent 2006; 17: 10-18.

30 Song S, Han D, Qu H et al: EDA gene mutations underlie non-syndromic oligodontia. J Dent Res 2009; 88: 126-131.

31 Mues G, Griggs R, Hartung AJ et al: From ectodermal dysplasia to selective tooth agenesis. Am J Med Genet 2009, e-pub ahead of print 5 June 2009; doi:10.1002/ ajmg.a.32801.

32 Colosimo PF, Hosemann KE, Balabhadra S et al: Widespread parallel evolution in sticklebacks by repeated fixation of ectodysplasin alleles. Science 2005; 307 : 1928-1933.

33 Zonana J, Elder ME, Schneider LC et al: A novel X-linked disorder of immune deficiency and hypohidrotic ectodermal dysplasia is allelic to Incontinentia Pigmenti and due to mutations in IKK-gamma (NEMO). Am J Hum Genet 2000; 67: 1555-1562.

34 Casal ML, Lewis JR, Mauldin EA et al: Significant correction of disease after postnatal administration of recombinant ectodysplasin A in canine X-linked ectodermal dysplasia. Am J Hum Genet 2007; 81: 1050-1060. 